\title{
A Stochastic Robust Optimization Approach for the Bidding Strategy of an Electric Vehicle Aggregator
}

\author{
Luis Baringo ${ }^{a, *}$, Raquel Sánchez-Amaro ${ }^{a}$ \\ ${ }^{a}$ Department of Electrical Engineering, Universidad de Castilla-La Mancha. Edificio \\ Politécnico, Avda. Camilo José Cela s/n, 13071 Ciudad Real, Spain
}

\begin{abstract}
This paper analyzes the bidding strategy problem of an electric vehicle aggregator that participates in the day-ahead energy market. The problem is formulated using a stochastic robust optimization model in which uncertainties in the day-ahead market prices and in the driving requirements of electric vehicles are modeled using scenarios and confidence bounds, respectively. The output of the proposed model is used to build the bidding curves to be submitted by the aggregator to the day-ahead market. We assume that the electric vehicle aggregator behaves as a price-taker in this market. A case study is analyzed to illustrate the main features of the proposed approach, as well as its applicability. We also compare the results with those achieved by considering other strategies. Results show that the proposed approach allows the aggregator to reduce the charging costs in comparison with other charging strategies. Moreover, the solution obtained is robust in the sense that driving requirements of electric vehicle users are met.
\end{abstract}

Keywords: Aggregator, electric vehicles, robust optimization, stochastic programming, strategic bidding, uncertainty

\footnotetext{
${ }^{*}$ Corresponding author

Email address: Luis.Baringo@uclm.es (Luis Baringo)
} 


\section{Notation}

2 The main notation used in this paper is stated below for quick reference, 3 while other symbols are defined as needed throughout the text. A subscript ${ }_{4} t / \omega$ in the symbols below denotes their values in the $t$ th time period / $\omega$ th 5 scenario.

6 Indices:

$7 \quad$ Time periods.

${ }_{8} \omega$ Scenarios.

9 Parameters:

${ }_{10} E^{\mathrm{A}} / E^{\mathrm{D}} \quad$ Energy contribution / drop of EVs arriving to / departing

11

${ }_{12} E_{t_{0}}$

13

${ }_{14} \underline{E}^{\max } / \bar{E}^{\max }$

15

${ }_{16} \underline{E}^{\min } / \bar{E}^{\min }$

17

${ }_{18} \underline{P}^{+, \max } / \bar{P}^{+, \max }$

19

${ }_{20} \underline{P}^{-, \max } / \bar{P}^{-, \max }$

21

${ }_{22} \Delta t$

${ }_{23} \eta^{+} / \eta^{-}$

24 from the EV aggregation [kWh].

Initial energy content of the virtual battery representing the EV aggregation [kWh].

Lower / upper bound for the maximum energy content of the virtual battery representing the EV aggregation [kWh].

Lower / upper bound for the minimum energy content of the virtual battery representing the EV aggregation [kWh].

Lower / upper bound for the maximum charging power of the virtual battery representing the EV aggregation $[\mathrm{kW}]$.

Lower / upper bound for the maximum discharging power of the virtual battery representing the EV aggregation $[\mathrm{kW}]$.

Time-step duration $[\mathrm{h}]$.

Charging / discharging efficiency of the virtual battery representing the EV aggregation [\%]. 


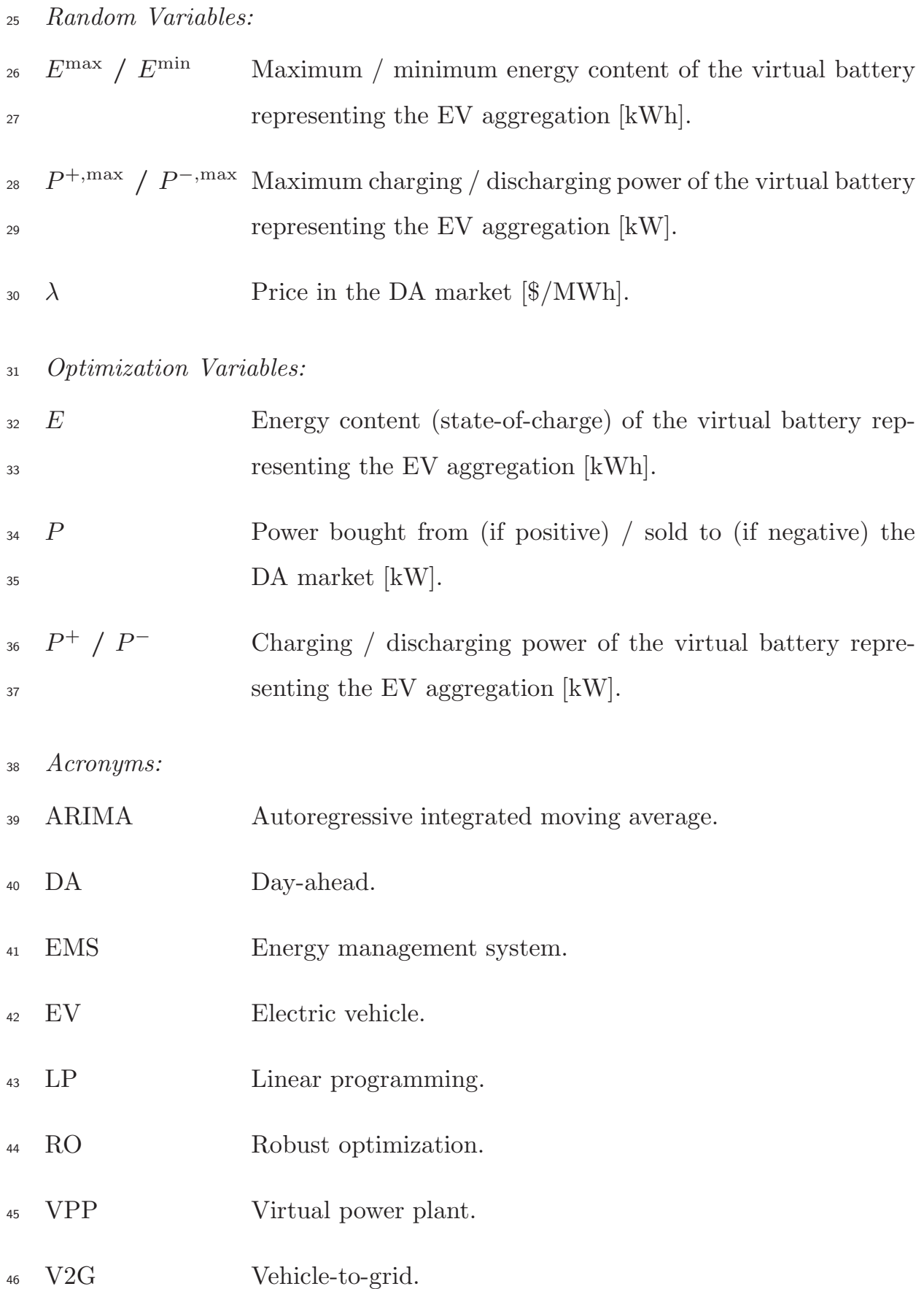




\section{Introduction}

48 1.1. Aim and Approach

The number of electric vehicles (EVs) is expected to increase in the near future due to, mainly, two reasons. First, the maturity of their technology makes EVs available for long-distance trips, which was one of the main drawbacks in the past. Second, due to the reduction of $\mathrm{CO}_{2}$ emissions and other greenhouse emissions if renewable energy sources are used to supply EVs [1]. This has motivated the use of incentive policies by some countries to encourage consumers ${ }_{55}$ to buy EVs instead of conventional vehicles, e.g., in Norway [2].

A large penetration of EVs supposes a big challenge. Traditional charging strategies consider that EV owners start charging their EVs as soon as they are parked and until the next trip or until the battery is full. In this sense, note

However, EVs are parked and available for charging most of the time. Thus, that most of the population arrive at home/work at similar times. Thus, most EVs are expected to charge at the same time. If the number of EVs is very large, this may translate in a peak in the energy consumption at these times. it would be possible to shift its charging to the most suitable time, e.g., to a time with low demand or with low prices. This would reduce the peak energy and/or would allow EV users to reduce their charging costs. Nevertheless, managing the charging of every individual EV has two problems. On the one hand, the limited flexibility of an individual EV. On the other hand, the high computation effort to deal with the management of a very large number of EVs. To overcome these two issues, it is generally convenient to use the figure of an aggregator, which can be seen as an energy management system (EMS) [3, 4] in charge of an EV fleet. The aggregator manages the charging of these EVs and ensures that the driving requirements of EV owners are met.

The figure of an EV aggregator has been proposed and considered in the technical literature using different approaches, e.g., considering unidirectional [5, 6] or bidirectional charging [7, 8]. This bidirectional charging is usually known as vehicle-to-grid (V2G) and allows the EV aggregator to behave as a 
77 producer or as a consumer [9] depending on the market prices and the driving

78 requirements.

Among the different problems faced by an EV aggregator, in this paper we focus on the problem of determining its bidding strategy in an energy mar-

81 ket. In particular, we determine the bidding strategy of an EV aggregator that ${ }_{82}$ participates in a day-ahead (DA) energy market.

A key point at the time of determining the bidding strategy of the EV 84 aggregator is the modeling the different sources of uncertainty that condition

85 this bidding strategy. These uncertainties include the market prices and the

${ }_{86}$ driving requirements of EV owners. To do so, in this paper we use scenarios to model the uncertainty in the market prices. This allows us to use a stochastic 88 programming model [10]. On the other hand, we use confidence bounds to

89 model the uncertainty in the driving requirements. This allows us to use robust 90 optimization (RO) [11]. Thus, the bidding strategy problem is formulated as a 91 hybrid stochastic robust model.

\subsection{Literature Review}

The technical literature about bidding strategy problems is extensive. Many

94 methods have been proposed for different entities, e.g., conventional power pro95 ducers [12], demand-response aggregators 13], virtual power plants (VPP) 14], 96 microgrids [15]. 12] proposes a bi-level model that allows representing the in${ }_{97}$ fluence of the power producer on market prices. 13 compares stochastic and 98 robust models for a demand-response aggregator. [14] proposes a stochastic 99 model for a VPP participating in both energy and reserve markets. [15] pro100 poses a hybrid stochastic robust model for a microgrid participating in the DA 101 and real-time energy markets.

Besides these methods, different approaches to deal with the bidding problem of an EV aggregator have been proposed, e.g., 16, 17, 18, 19, 20, 21, 22, 23].

Some of these methods consider the participation of EVs in both energy and capacity markets. [16] and [17] consider the participation of the EV aggregator in the DA and reserve markets. [18] proposes a stochastic approach 
and considers the participation of the EV aggregator in the DA and regulation markets. 19] analyzes the optimization of charging and frequency regulation capacity bids using a stochastic dynamic programming model. The problem is formulated in this case using a Markov decision problem. 20] proposes a stochastic mathematical programming with equilibrium constraints problem for the participation of EV aggregators in the DA and ancillary services markets.

Other methods consider the participation of EVs only in energy markets. [21] and [22] formulate the bidding problem in the DA market using a bi-level model, which explicitly represents the clearing of the DA market. [23] models the risk associated with the bidding decisions in the DA market using the conditional value-at-risk.

However, none of the above methods for EV bidding considers a robust approach. In these references, uncertainties are generally modeled using scenarios. Thus, the driving requirements of EVs may not be met if these scenarios are not properly generated.

Note that both [21] and this paper deal with the same problem, namely, the bidding strategy of an EV aggregator in the DA market. However, these two works have three main differences: i) 21] considers that the EV aggregator is a price-maker while in this paper it is considered as a price-taker; ii) uncertainties in [21] are modeled using scenarios, while in this paper we model uncertainties using both scenarios and confidence bounds; and iii) 21] proposes a stochastic model, while in this paper we describe a hybrid stochastic robust model.

\subsection{Contributions}

The main contribution of this paper is to provide a new approach based on a hybrid stochastic robust model for the bidding strategy of an EV aggregator in the DA market.

On the one hand, stochastic programming has been widely used for offering strategy problems [9, 12]. On the other hand, RO has been recently used in offering and bidding strategy problems of conventional units [24], concentrating solar power plants [25], and VPPs [26], since it constitutes a flexible tool that 
can be easily included in an EMS, e.g., in an EV aggregator in charge of an EV fleet.

However, to the best of our knowledge, there is no paper in the technical literature that combine stochastic programming and robust optimization for the bidding problem of an EV aggregation.

The advantage of the method proposed in this paper over other methods based solely on stochastic programming, e.g., [14], is that driving requirements are generally difficult to forecast. Thus, a very large number of scenarios would be needed to obtain an accurate representation of the uncertainty in the driving requirements. This may result in a very computationally complex model.

On the other hand, the advantage of this method in comparison with other methods based solely on RO, e.g., [24, 26], is that these methods are usually too conservative. Moreover, there are many methods that provide good forecasts of market prices. Thus, the number of scenarios needed to obtain an accurate representation of the uncertainty in the market prices is not that large as in the case of the driving requirements.

As in this paper, [13] and [15] analyze a combination of stochastic and robust models for the bidding problem of a demand response aggregator and a microgrid, respectively. However, in [13] both models are analyzed independently. On the other hand, [15] uses confidence bounds to model uncertainty in real-time market prices, and scenarios to model uncertainty in DA market price scenarios and wind-power production. Note that while prices appear in the objective function of the optimization problem described in [15], the wind-power production appears within the constraints. Thus, this may result in suboptimal results or even in an infeasible solution if the actual wind-power production (or the driving requirements in the case of this paper) is different to one of the scenarios considered.

As a summary and given the above context, the contributions of this paper are fourfold:

1. To propose a novel approach for the bidding strategy in the DA market of 
an EV aggregator that faces uncertain market prices and uncertain driving patterns.

2. To re-formulate the problem in 1 as a stochastic robust problem.

3. To use the outputs of problem in 2 to build the bidding curves of the EV aggregator.

4. To provide and analyze the results of a case study.

\subsection{Paper Organization}

The remainder of the paper is organized as follows. Section 2 describes the main features of the bidding strategy problem. Section 3 provides the model of an EV aggregator that manages an EV fleet. The characterization of the different sources of uncertainties is described in Section 4 . The bidding problem is formulated in Section 5 using a deterministic approach, which is extended in Section 6 using a stochastic robust approach that takes into account the uncertainties in the market prices and in the driving requirements. Section 7 provides the results of a case study. Section 8 concludes the paper with some relevant remarks.

\section{Problem Description}

We consider a number of EVs within an EV fleet. These EVs are managed by an EV aggregator that works as an EMS. The individual EVs communicate the aggregator their driving requirements, and, then, the aggregator determines the optimal charging time of each $\mathrm{EV}$ with the aim of minimizing the overall charging costs but, at the same time, meeting the driving requirements of $\mathrm{EV}$ users. We assume that charging points and EVs are equipped with the V2G technology so that EVs can be also used to supply energy to the grid if needed.

In order to supply the EVs, the aggregator participates in the DA energy market. To do so, instead of considering each EV individually, the aggregator considers the aggregation of all EVs in the fleet [8]. Then, considering the driving requirements of the EVs in the fleet, the aggregator decides the bidding 
strategy and sends these bidding decisions to the market operator. With these bidding decisions, as well as with the offering and bidding decisions of other market participants, the market operator clears the DA market and communicates the aggregator the market outputs.

This problem structure is schematically represented in Fig. 1.

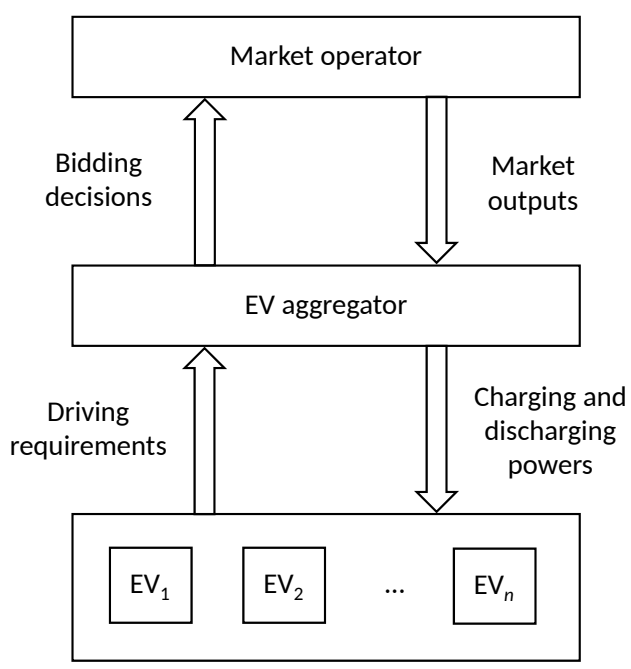

Figure 1: Problem structure.

We consider a price-taker approach that assumes that the EV aggregator cannot affect market prices. If the size of the EV fleet managed by the aggregator is sufficiently large, it may be able to alter market prices. In such a case, it is necessary to represent the clearing of the market within the optimization problem so that the resulting problem is a mathematical program with equilibrium constraints [27] and the models proposed in [21] and [22] may be considered. However, in this paper we assume that the size of the EV fleet is not that large.

The problem is solved for a planning horizon of one day. For the sake of simplicity, the planning horizon is discretized into 24 hourly time periods. However, note that the duration of these time steps may be reduced in order to obtain a detailed modeling of the charging process. This is out of the scope of 
this paper and will be subject of future research.

\section{EV Aggregator}

We consider an aggregation of EVs managed by the so-called EV aggregator. The EV aggregation is represented by a virtual battery that takes into account the driving patterns of EV users, the physical characteristics of the individual EV batteries, as well as the physical characteristics of the charging infrastructure. To do so, we use the model proposed in [8], which comprises the following equations:

$$
\begin{aligned}
& E_{t}=E_{t-1}+P_{t}^{+} \Delta t \eta^{+}+E_{t}^{\mathrm{A}}-\frac{P_{t}^{-} \Delta t}{\eta^{-}}-E_{t}^{\mathrm{D}} \quad \forall t \\
& E_{t_{\mathrm{f}}} \geq E_{t_{0}} \\
& P_{t}=P_{t}^{+}-P_{t}^{-} \quad \forall t \\
& 0 \leq P_{t}^{+} \leq P_{t}^{+, \max } \quad \forall t \\
& 0 \leq P_{t}^{-} \leq P_{t}^{-, \max } \quad \forall t \\
& E_{t}^{\min } \leq E_{t} \leq E_{t}^{\max } \quad \forall t .
\end{aligned}
$$

Constraints (11) represent the energy balance of the virtual battery representing the EV aggregation. The energy content of the virtual battery at each time period is equal to the energy content in the previous time period, the charging energy, and the energy contributions of EVs arriving to the EV aggregation, minus the discharging energy and the energy drops of EVs departing from the EV aggregation at that time period. Constraint (2) imposes a lower bound on the energy stored in the virtual battery at the end of the planning horizon. If this constraint is not included, the battery would tend to be depleted over the planning horizon. Constraints (3) define the power that is sold to / bought from the DA market as the charging minus the discharging powers. Note that we assume that EVs can both charge and discharge if it is economically profitable, i.e., we assume that EVs are equipped with V2G technology. Finally, constraints (4), 
(5]), and (6) impose bounds on the charging power, the discharging power, and the energy content of the virtual battery, respectively.

We consider two different variables for the charging and discharging of the EV aggregation, respectively. Thus, binary variables to avoid charging and discharging at the same time would be needed. However, we also consider charging and discharging efficiencies. Since these constraints will be used in an optimization problem that aims at minimizing / maximizing the charging / discharging costs / profits, it is not optimal to charge and discharge at the same time. Thus, it is not necessary to include these binary variables. Note that this assumption is valid provided that charging and discharging efficiencies are not 1. Note also that not including such binary variables allows us to reduce the computation burden of the proposed approach.

The power and energy bounds in constraints (4), (5), and (6) are obtained by considering the driving requirements of EV users, as well as the physical characteristics of the EV fleet and the charging points. Additional details about how these bounds are computed are provided in [8].

\section{Uncertainty Characterization}

The bidding decisions in the DA market must be made one day in advance and for all the hours of the following day. At that time, the EV aggregator does not know neither the DA market prices nor the driving requirements of the EV fleet. Thus, in order to obtain informed bidding decisions, it is necessary to represent the uncertainty in these variables.

There are generally two ways of representing uncertainties within this type of problems. One is to use a set of scenarios that model different realizations of the uncertain variables. The other option is to consider that uncertain variables take values within known confidence bounds. In this paper, we use a combination of both alternatives.

On the one hand, we use a set of scenarios indexed by $\omega$ to model the uncertainty in the DA market prices. These scenarios are obtained using historical 

is:

data of DA market prices and the ARIMA model described in [28].

While there are many methods in the technical literature to generate accurate DA market price scenarios, this is not the case for driving requirements. Thus, instead of scenarios, we use confidence bounds to model the uncertainty in the driving requirements, namely, in the maximum charging power, in the minimum discharging power, as well as in the minimum and maximum energy content of the virtual battery. We assume that these power and energy limits are not known at the time the bidding decisions in the DA market are made; however, we know that they take values within known confidence bounds. That

$$
\begin{aligned}
& \underline{P}_{t}^{+, \max } \leq P_{t}^{+, \max } \leq \bar{P}_{t}^{+, \max } \quad \forall t \\
& \underline{P}_{t}^{-, \max } \leq P_{t}^{-, \text {max }} \leq \bar{P}_{t}^{-, \max } \quad \forall t \\
& \underline{E}_{t}^{\min } \leq E_{t}^{\min } \leq \bar{E}_{t}^{\min } \quad \forall t \\
& \underline{E}_{t}^{\max } \leq E_{t}^{\max } \leq \bar{E}_{t}^{\max } \quad \forall t .
\end{aligned}
$$

The above upper and lower power and energy limits may be obtained using historical data to forecast the future driving requirements. In this paper, we simply take the data provided in [8] and consider that power and energy limits vary within $\pm 5 \%$ of their expected values. An accurate modeling of these confidence bounds is out of the scope of this paper.

It is important to note that the uncertainty in the driving requirements decreases as we increase the number of EVs managed by the EV aggregator as shown in [22].

The advantage of using confidence bounds to model the uncertainty in the driving requirements is that the solution would be feasible for every realization of the uncertain power and energy limits within the considered bounds. If, instead, scenarios are used to model these uncertainties, then, the solution may be infeasible if the actual realizations of the power and energy limits are different from the considered scenarios. Moreover, it is generally easier to obtain 
confidence bounds than generating accurate scenarios.

The above uncertainty representation allows us to formulate the bidding problem as a stochastic robust model. It is stochastic [10] from the point of view of market prices and robust [11] from the point of view of power and energy bounds.

Finally, note that the market price scenarios and the confidence bounds for driving requirements described above are considered as input data of the model formulated in the following section. This model is general and other methods may be considered to obtain more accurate scenarios and confidence bounds.

\section{Deterministic Formulation}

The self-scheduling problem of an EV aggregator that participates in the DA market can be formulated using the linear programming (LP) model below: $\min _{\Theta_{t}}$

$$
\sum_{t} \lambda_{t} P_{t} \Delta t
$$

subject to

$$
\text { Constraints (11) }-(6) \text {, }
$$

where variables in set $\Theta_{t}=\left\{P_{t}, P_{t}^{+}, P_{t}^{-}, E_{t}\right\}, \forall t$, are the optimization variables of problem (11)-(12).

The objective function (11) represents the minimization of the costs of purchasing energy in the DA market. Note that if the EV aggregator sells power to the market in time period $t$, then $P_{t}<0$ and these costs are negative, i.e., in this case term $\lambda_{t} P_{t} \Delta t$ represents the minus profit achieved by the EV aggregator.

Constraints of problem (11)-(12) include the constraints of the virtual battery used to represent the EV aggregation as described in Section 3 .

Problem (11)-(12) allows the EV aggregator to determine the charging and discharging powers at each time period that minimize its charging costs over the planning horizon and meet the driving requirements of the EV fleet. However, 
it is important to note that at the time this problem is solved by the EV aggregator, it does not know neither the values of the market prices nor the driving requirements of the EV users. Thus, it is necessary to model this uncertainty as explained in Section 4. This uncertainty representation allows us to formulate the stochastic robust model described in the following section.

Note that by solving problem (11)-(12) we obtain the aggregated charging and discharging powers of the EV aggregator. Then, in a second step, the aggregator should distribute these powers among the individual EVs. This second step is out of the scope of this paper.

\section{Stochastic Robust Formulation}

The problem of determining the bidding strategy in the DA market of an EV aggregator that faces uncertain market prices and driving requirements can be formulated using the stochastic robust model below:

$$
\min _{\Theta_{\omega, t}, \Theta_{\omega, t}^{\mathrm{R}}}
$$

$$
\sum_{\omega} \pi_{\omega} \sum_{t} \lambda_{\omega, t} P_{\omega, t} \Delta t
$$

subject to

$$
\begin{aligned}
& E_{\omega, t}=E_{\omega, t-1}+P_{\omega, t}^{+} \Delta t \eta^{+}-\frac{P_{\omega, t}^{-} \Delta t}{\eta^{-}}+E_{t}^{\mathrm{A}}-E_{t}^{\mathrm{D}} \quad \forall \omega, t \\
& E_{\omega, t_{\mathrm{f}}} \geq E_{t_{0}} \quad \forall \omega \\
& P_{\omega, t}=P_{\omega, t}^{+}-P_{\omega, t}^{-} \quad \forall \omega, t \\
& P_{\omega, t} \leq P_{\tilde{\omega}, t} \text { if } \lambda_{\omega, t} \geq \lambda_{\tilde{\omega}, t} \quad \forall \omega, \tilde{\omega}, t \\
& P_{\omega, t}^{+}+\Gamma_{\omega, t}^{\mathrm{P}+} v_{\omega, t}^{\mathrm{P}+}+\mu_{\omega, t}^{\mathrm{P}+} \leq \frac{1}{2}\left(\bar{P}_{t}^{+, \max }+\underline{P}_{t}^{+, \max }\right) \quad \forall \omega, t \\
& v_{\omega, t}^{\mathrm{P}+}+\mu_{\omega, t}^{\mathrm{P}+} \geq \frac{1}{2}\left(\bar{P}_{t}^{+, \max }-\underline{P}_{t}^{+, \max }\right) y_{\omega, t}^{\mathrm{P}+} \quad \forall \omega, t \\
& 1 \leq y_{\omega, t}^{\mathrm{P}+} \quad \forall \omega, t \\
& P_{\omega, t}^{-}+\Gamma_{\omega, t}^{\mathrm{P}-} v_{\omega, t}^{\mathrm{P}+}+\mu_{\omega, t}^{\mathrm{P}-} \leq \frac{1}{2}\left(\bar{P}_{t}^{-, \max }+\underline{P}_{t}^{-, \max }\right) \quad \forall \omega, t \\
& v_{\omega, t}^{\mathrm{P}-}+\mu_{\omega, t}^{\mathrm{P}-} \geq \frac{1}{2}\left(\bar{P}_{t}^{-, \max }-\underline{P}_{t}^{-, \max }\right) y_{\omega, t}^{\mathrm{P}-} \quad \forall \omega, t
\end{aligned}
$$




$$
\begin{aligned}
& 1 \leq y_{\omega, t}^{\mathrm{P}-} \quad \forall \omega, t \\
& E_{\omega, t}+\Gamma_{\omega, t}^{\mathrm{Emax}} v_{\omega, t}^{\mathrm{Emax}}+\mu_{\omega, t}^{\mathrm{Emax}} \leq \frac{1}{2}\left(\bar{E}_{t}^{\mathrm{max}}+\underline{E}_{t}^{\max }\right) \\
& v_{\omega, t}^{\mathrm{Emax}}+\mu_{\omega, t}^{\mathrm{Emax}} \geq \frac{1}{2}\left(\bar{E}_{t}^{\max }-\underline{E}_{t}^{\max }\right) y_{\omega, t}^{\mathrm{Emax}} \quad \forall \omega, t \\
& 1 \leq y_{\omega, t}^{\mathrm{Emax}} \quad \forall \omega, t \\
& -E_{\omega, t}+\Gamma_{\omega, t}^{\mathrm{Emin}} v_{\omega, t}^{\mathrm{Emin}}+\mu_{\omega, t}^{\mathrm{Emin}} \leq-\frac{1}{2}\left(\bar{E}_{t}^{\min }+\underline{E}_{t}^{\min }\right) \quad \forall \omega, t \\
& v_{\omega, t}^{\mathrm{Emin}}+\mu_{\omega, t}^{\mathrm{Emmin}} \geq \frac{1}{2}\left(\bar{E}_{t}^{\min }-\underline{E}_{t}^{\min }\right) y_{\omega, t}^{\mathrm{Emin}} \quad \forall \omega, t \\
& 1 \leq y_{\omega, t}^{\mathrm{Emin}} \quad \forall \omega, t \\
& v_{\omega, t}^{\mathrm{P}+}, \mu_{\omega, t}^{\mathrm{P}+}, y_{\omega, t}^{\mathrm{P}+}, v_{\omega, t}^{\mathrm{P}-}, \mu_{\omega, t}^{\mathrm{P}-}, y_{\omega, t}^{\mathrm{P}-}, v_{\omega, t}^{\mathrm{Emax}}, \mu_{\omega, t}^{\mathrm{Emax}}, y_{\omega, t}^{\mathrm{Emax}}, v_{\omega, t}^{\mathrm{Emin}}, \mu_{\omega, t}^{\mathrm{Emin}}, y_{\omega, t}^{\mathrm{Emin}} \geq 0 \\
& \forall \omega, t,
\end{aligned}
$$

where variables in sets $\Theta_{\omega, t}=\left\{P_{\omega, t}, P_{\omega, t}^{+}, P_{\omega, t}^{-}, E_{\omega, t}\right\}, \forall \omega, t$, and $\Theta_{\omega, t}^{\mathrm{R}}=\left\{v_{\omega, t}^{\mathrm{P}+}\right.$, $\left.\mu_{\omega, t}^{\mathrm{P}+}, v_{\omega, t}^{\mathrm{P}-}, y_{\omega, t}^{\mathrm{P}+}, \mu_{\omega, t}^{\mathrm{P}-}, y_{\omega, t}^{\mathrm{P}-}, v_{\omega, t}^{\mathrm{Emax}}, \mu_{\omega, t}^{\mathrm{Emax}} y_{\omega, t}^{\mathrm{Emax}}, v_{\omega, t}^{\mathrm{Emin}}, \mu_{\omega, t}^{\mathrm{Emin}}, y_{\omega, t}^{\mathrm{Emin}}\right\}, \forall \omega, t$, are the optimization variables of problem (13)-(30). Variables in sets $\Theta_{\omega, t}^{\mathrm{R}}, \forall \omega$, $t$, are auxiliary variables needed to derive the robust problem [11].

Problem (13)-(30) is an LP problem. LP problems can be solved using available optimization software, e.g., CPLEX [29], and its convergence to the optimum is guaranteed [30].

The objective function (13) represents the minimization of the expected costs of participating in the DA market. Note that, as in problem (11)-(12), $P_{\omega, t}$ can also take both positive (if charging) and negative (if discharging) values. In the latter, terms $\lambda_{\omega, t} P_{\omega, t} \Delta t$ represent the minus income achieved by the EV aggregator. As a difference with the objective function of the deterministic problem (11), here the costs for each scenario are multiplied by the corresponding probability.

Constraints (14)-(16) are similar to constraints (2)-(4) of the deterministic problem; however, these constraints are considered for each scenario in this case. Constraints (17) ensure that the bidding curves decrease monotonically. Constraints (18)-(20), (21)-(23), (24)-(26), and (28)-(30) are robust constraints that allow us to consider the uncertainty in the power and energy bounds, i.e., 
in random variables $P_{t}^{+, \max }, P_{t}^{-, \max }, E_{t}^{\max }$, and $E_{t}^{\min }$, respectively. Additional details on how to obtain these constraints are provided in the Appendix of this paper and in [11].

In model (13)-(30) we include control parameters $\Gamma_{\omega, t}^{\mathrm{P}+}, \Gamma_{\omega, t}^{\mathrm{P}-}, \Gamma_{\omega, t}^{\mathrm{Emax}}$, and $\Gamma_{\omega, t}^{E m i n}$ in constraints (18), (21), (24), and (28), respectively. These parameters can take values between 0 and 1 , and allow us to control the level of robustness in the problem. If one of these parameters is fixed to 0 , then we ignore the uncertainty in the random variable of the corresponding constraint. However, if it is fixed to 1 , then we consider that the uncertain variable in that constraint can take any value within the considered confidence bound and, thus, we obtain a comparatively more robust solution. The output of model (13)-(30), i.e., the bidding strategy of the EV aggregator, depends on the values considered for these parameters.

Note that in problem (13)-(30), we decide the charging / discharging power $P_{\omega, t}$ for each scenario, i.e., variable $P_{\omega, t}$ depends on the scenario realization $\omega$. However, note that these decisions must be made before the EV aggregator knows the actual scenario realization. Nevertheless, we relax the nonanticipativity constraints that should impose $P_{\omega, t}=P_{\tilde{\omega}, t}, \forall \omega, \tilde{\omega}$, and use these scenario-dependent variables and the corresponding market price for each scenario and time period to build the bidding curves to be submitted by the EV aggregator to the market operator.

Finally, note that the bidding strategy problem proposed in this paper may be applied not only by an EV aggregator but also by other entities, e.g., the EMS in charge of a VPP, if the corresponding components are modeled within the proposed approach.

\section{Case Study}

The stochastic robust model (13)-(30) is analyzed in this section using a realistic case study. 


\subsection{Data}

The bidding strategy is decided for a planning horizon of 24 hours divided into hourly time steps.

We consider an aggregation of EVs whose main characteristics are provided in Table 1 For the sake of simplicity, we assume that all EVs in the aggregation have the same characteristics. Therefore, the charging and discharging efficiencies of the EV aggregation are equal to the individual charging and discharging efficiencies provided in Table 1 .

Table 1: Data of EVs in the aggregation

\begin{tabular}{cc}
\hline \multicolumn{2}{c}{ Table 1: Data of EVs in the aggregation } \\
\hline Number of EVs & 1200 \\
\hline Capacity of batteries & $20 \mathrm{kWh}$ \\
\hline Maximum charging power & $5 \mathrm{~kW}$ \\
\hline Maximum discharging power & $5 \mathrm{~kW}$ \\
\hline Charging efficiency & $90 \%$ \\
\hline Discharging efficiency & $90 \%$ \\
\hline Initial energy content in the virtual battery & $18000 \mathrm{kWh}$ \\
\hline
\end{tabular}

The driving patterns of this EV aggregation are based on the data provided in [8]. Figs. 2 and 3 provide the energy and power bounds of the virtual battery, respectively. Note that in these figures we represent the confidence bounds needed to solve the stochastic robust model (13)-(30). To obtain these bounds, we take the data provided in [8] and consider that power and energy limits vary within $\pm 5 \%$ of their expected values. Finally, the energy contributions / drops of EVs arriving to / departing from the virtual battery at each time step are provided in Fig. 4. These data are also based on the data provided in [8].

We consider that the EV aggregator participates in the New England electricity market [31]. We use historical data of DA market prices and the ARIMA model proposed in [28] to generate 20 scenarios for the 24 hours of May 4, 2011. These scenarios are represented in Fig. 5 and all of them have the same 


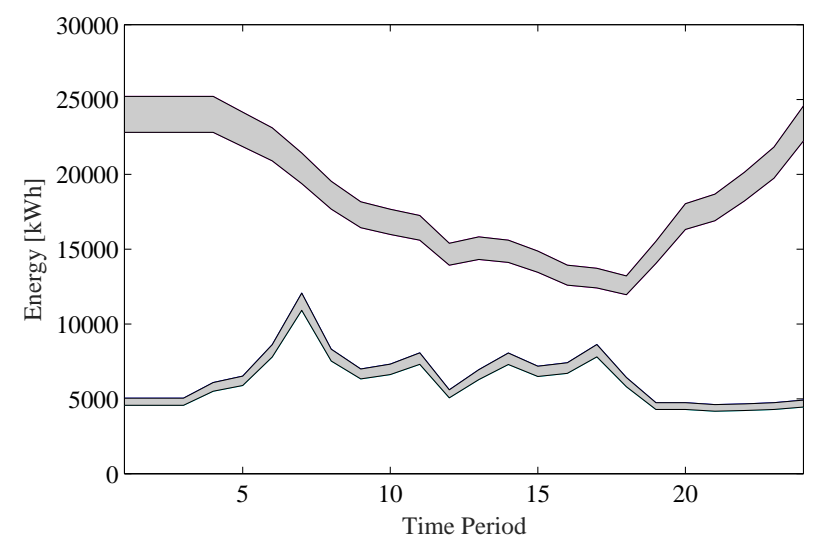

Figure 2: Energy bounds.

probability.

\subsection{Results}

First, we fix the value of the control parameters $\Gamma_{\omega, t}^{\mathrm{P}+}, \Gamma_{\omega, t}^{\mathrm{P}-}, \Gamma_{\omega, t}^{\mathrm{Emax}}$, and $\Gamma_{\omega, t}^{\mathrm{Emin}}$ to $1, \forall \omega, t$. This allows us to obtain a conservative solution since we take into account that the uncertain power and energy bounds can take any value within the considered confidence bounds.

We solve problem (13)-(30) and obtain the bidding curves in hours 3, 11, and 21, which are depicted in Figs. 6, 7 and 8, respectively.

Market prices in hour 3 (during night) are comparatively low. Thus, the EV aggregator participates in the DA market as a demand in that hour, i.e., it is willing to charge the batteries of EVs. However, note that the bid power is 0 if the price is higher than $\$ 39.2 / \mathrm{MWh}$ in that hour. A different result can be observed in the bidding curve for hour 11. In this case, depending on the price, the EV aggregator participates in the DA market as a demand (positive values of power) or as a generating unit (negative values of power). This way, the aggregator takes advantage of price differences to sell part of the energy stored 403 in the virtual battery. Finally, we observe in Fig. 8 that the EV aggregator 404 behaves as an inelastic generating unit in hour 21. If we look at the market 


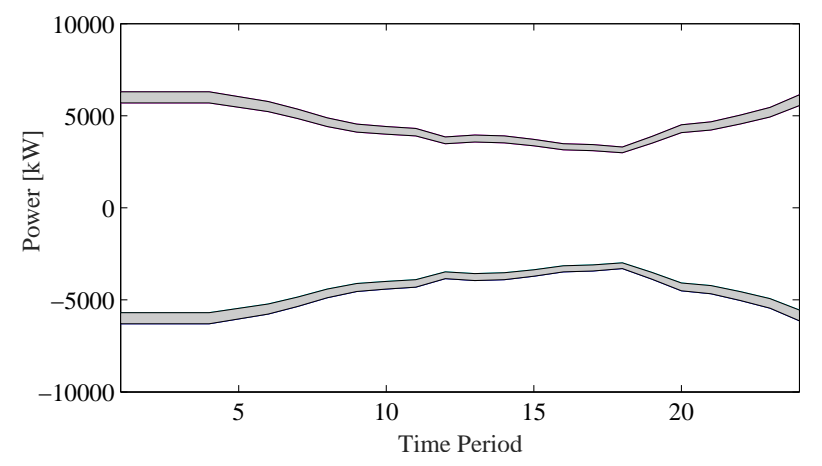

Figure 3: Power bounds.

405 price scenarios in Fig. 5, we observe that the market prices in hour 21 are 406 comparatively higher than those prices in the previous and following hours. Thus, the EV aggregator is willing to inject energy into the network, i.e., to ${ }_{408}$ discharge the EV fleet, in this hour. This results in an inelastic offer curve that 409 indicates that the EV aggregator is willing to sell $4222 \mathrm{~kW}$ to the DA market independently of the price.

\subsection{Impact of Robust Control Parameters}

Next, we analyze the impact of the control parameters on the bidding strategy. We solve problem (13)-(30) again for different values of these control pa- 


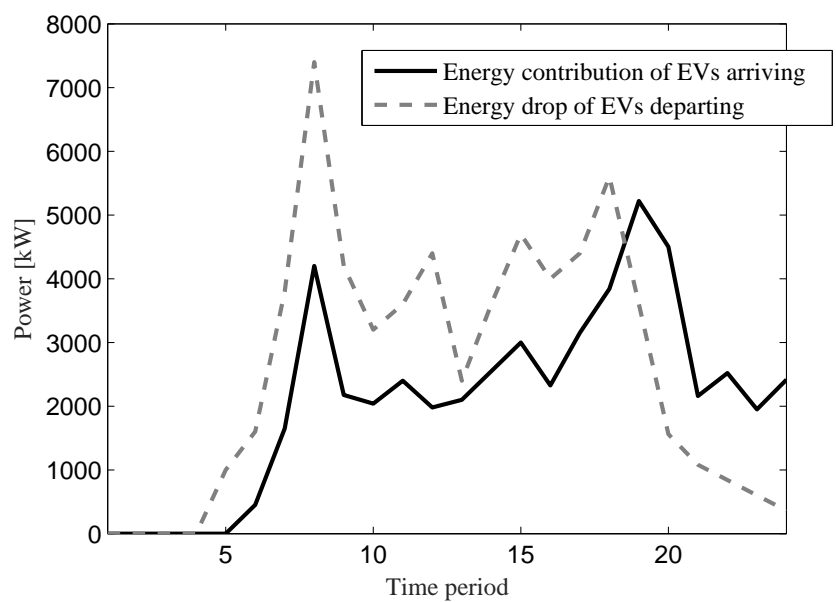

Figure 4: Energy contribution/drop of EVs arriving to/departing from the virtual battery.

424 on the expected operation costs. These costs for different values of the control 425 parameters are provided in Table 2. These costs are obtained as the average of 426 the 20 scenarios.

Table 2: Expected operation costs for different values of control parameters [\$]

\begin{tabular}{ccc}
$\Gamma=0$ & $\Gamma=0.5$ & $\Gamma=1$ \\
\hline 366.41 & 381.46 & 396.89 \\
\hline
\end{tabular}

${ }_{427}$ Considering the more robust solution, i.e., fixing all control parameters to ${ }_{428} 1$, results in an expected operation cost that is $8.32 \%$ higher than the expected ${ }_{429}$ cost if these parameters are fixed to 0, i.e., if a risky strategy is selected. How430 ever, note that if $\Gamma=0$, we ignore the uncertainty in the power and energy bounds, which are assumed to take the average values of the considered confi${ }_{432}$ dence bounds. Then, if a deviation in these power and energy bounds occurs, ${ }_{433}$ the EV aggregation should compensate it by participating in other market mech${ }_{434}$ anisms, e.g., the balancing market, which also has a cost. 


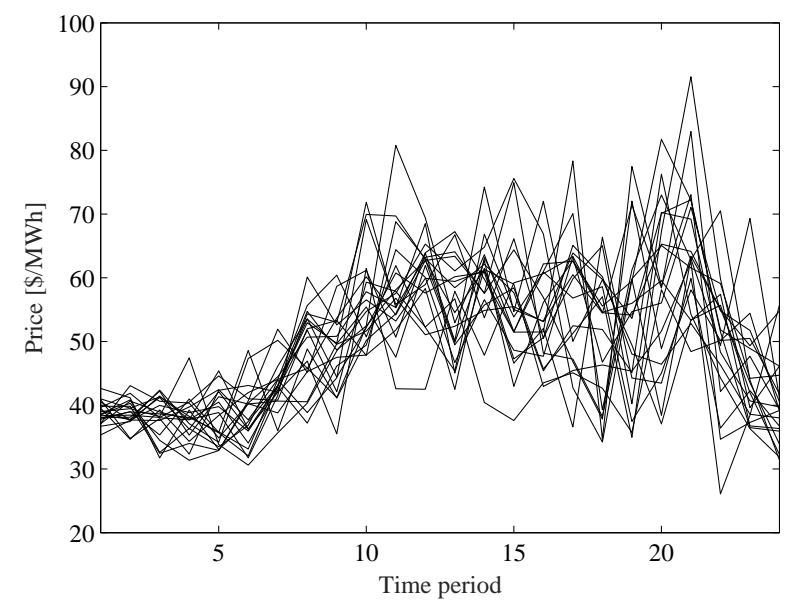

Figure 5: Price scenarios.

\subsection{Comparison of Different Charging Strategies}

Next, the results are compared with two benchmark charging strategies:

1. Unidirectional charging (no V2G): we solve problem (13)-(30) by considering that $P_{\omega, t}^{-}=0, \forall \omega, t$, i.e., we assume that the $\mathrm{EV}$ aggregator cannot sell the energy of the virtual battery in the market.

2. Conventional charging: instead of solving an optimization problem, we assume that the EV aggregator charges the virtual battery as much as possible, i.e., the virtual battery is charged at $P_{t}^{+, \max }$ given that $E_{\omega, t} \leq E_{t}^{\max }$. This corresponds to a conventional charging, when EVs start charging as soon as they are parked and until the battery is full or they depart for the next trip.

The bidding curves for hour 15 and for the different charging strategies are depicted in Fig. 11. We assume that the control parameters are fixed to 1.

Note that the bidding curves are different depending on the strategy selected. The larger charging power corresponds to the conventional charging. We also observe that for prices below $\$ 55.5 / \mathrm{MWh}$, considering the V2G technology re-

sults in a larger charging power than without the V2G technology. This can be 


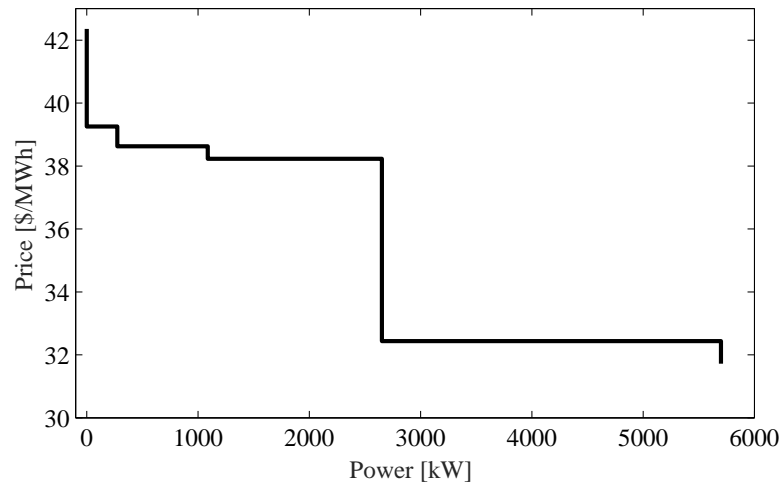

Figure 6: Bid curve in hour 3.

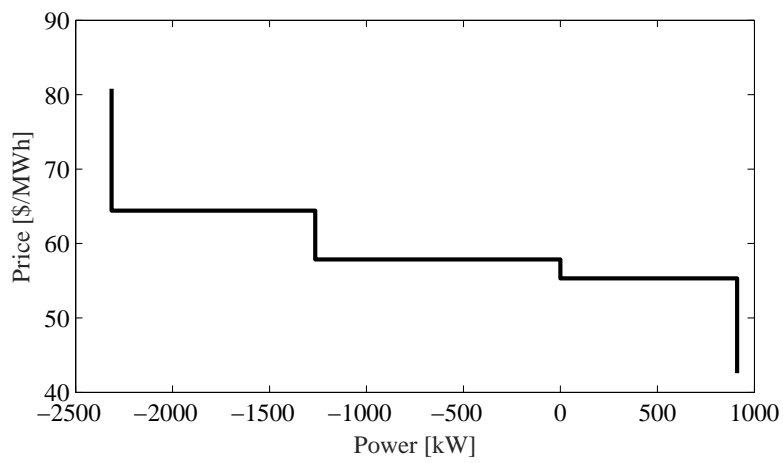

Figure 7: Bid curve in hour 11. 3.

explained by the fact that, if the $\mathrm{V} 2 \mathrm{G}$ technology is available, the $\mathrm{EV}$ aggregator prefers charging a larger energy in hour 15 in order to sell it in the following hours, when the prices are comparatively higher.

The expected costs for the different charging strategies are provided in Table

Note that the conventional charging results in the highest expected operation cost. This is so because EVs in the fleet start charging as soon as they are parked, independently of the market prices. On the other hand, if the V2G is available and the EV aggregator takes advantage of it, the expected operation 


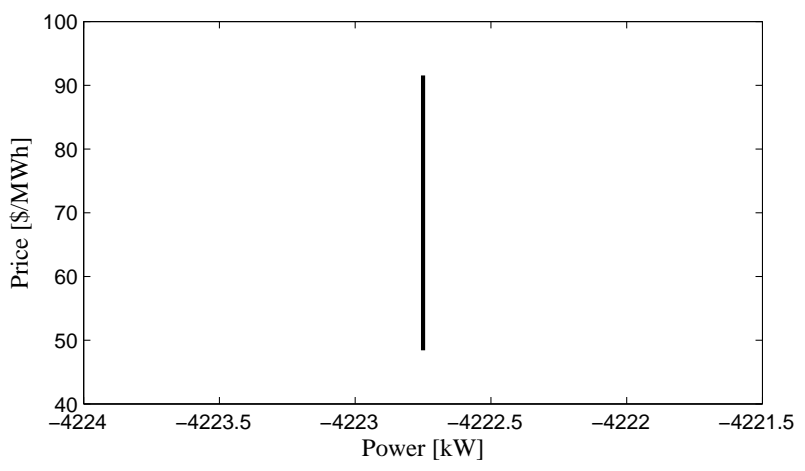

Figure 8: Bid curve in hour 21.

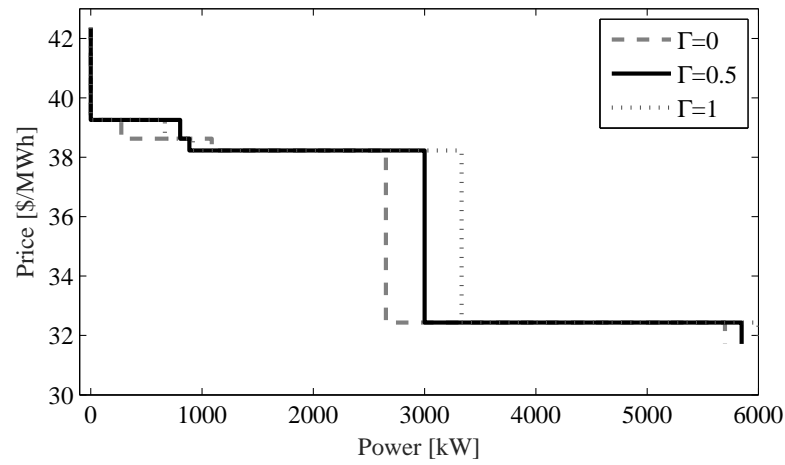

Figure 9: Bid curves in hour 3 for different values of $\Gamma$.

461 cost is reduced by $25.03 \%$ in comparison with the case without V2G. Considering

${ }^{462}$ the V2G technology, the EV aggregator can sell energy to the DA market in

${ }_{463}$ hours with comparatively high market prices (and is paid for that) and buy

464 energy from the DA market in hours with comparatively low prices.

465 7.5. Computation Burden of the Problem

${ }_{466}$ The LP model (13)-(30) was solved using CPLEX 12.2.0.1 [29] under GAMS

${ }_{467}$ [32] on an Intel-Core i3 processor clocking at $3.4 \mathrm{GHz}$ and $4 \mathrm{~GB}$ of RAM. The

${ }_{468}$ computation time required to obtain the optimal solution for different values

469 of the control parameters is less than $1 \mathrm{~s}$, which is compatible with the time 


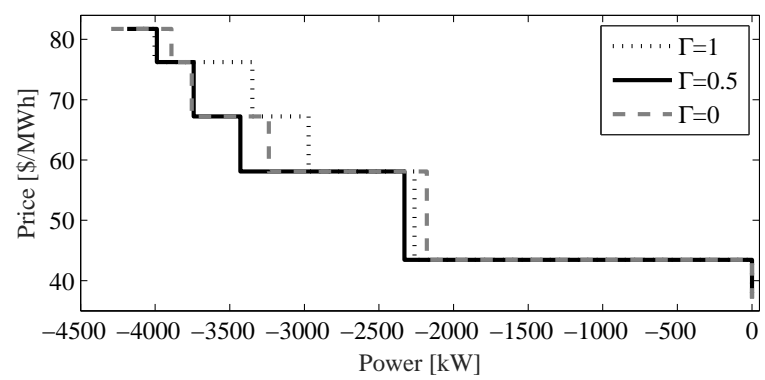

Figure 10: Bid curves in hour 20 for different values of $\Gamma$.

\begin{tabular}{ccc}
\multicolumn{3}{c}{ Table 3: Expected operation costs for different charging strategies [\$] } \\
\hline Proposed strategy & Without V2G & Conventional charging \\
\hline 396.89 & 529.38 & 851.08 \\
\hline
\end{tabular}

requirements for deciding the bidding strategy in the DA market.

Finally, we show in Figure 12 the dependency of the expected profit with the number of scenarios. To do so, we run the model again but considering an increasing number of scenarios. In this case, we consider that the control parameters are fixed to 0.5 .

We observe that there are important changes in the expected profit if the number of scenarios is low. However, the expected profit becomes approximately stable as we increase this number. Nevertheless, note that increasing the number of scenarios to run the model would not be a big deal. The resulting problem is an LP problem and there are many solvers that run this type of problems with a very large number of variables and constraints in almost no time. If needed, scenario reduction methods may be also considered [33].

\section{Conclusions}

A stochastic robust approach has been proposed for the bidding strategy problem of an EV aggregator. The conclusions below are in order: 


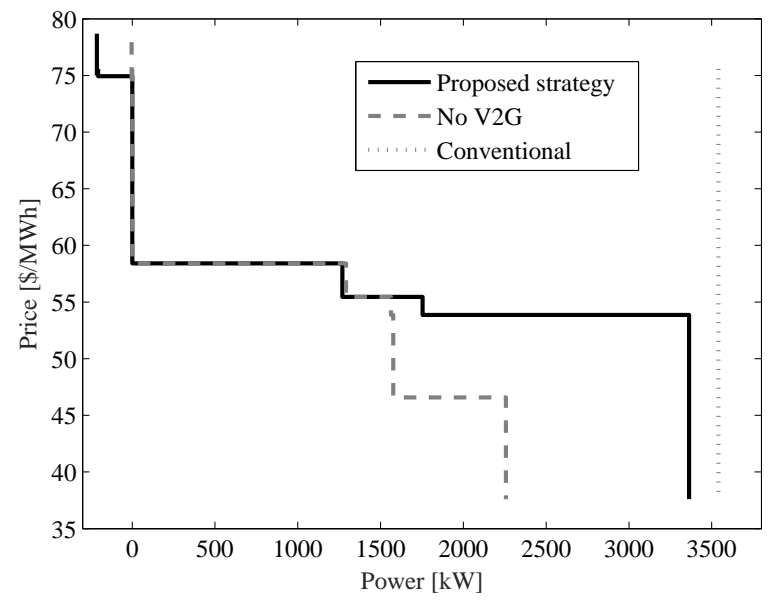

Figure 11: Bid curves in hour 15 for different charging strategies.

499 Section 5 .

1. An accurate modeling of the uncertainties in market prices and driving requirements is needed to obtain informed bidding decisions for the EV aggregator. In this sense, the proposed stochastic robust approach constitutes an appropriate tool.

2. It is possible to control the level of robustness in the solution by using the proposed approach.

3. The use of the $\mathrm{V} 2 \mathrm{G}$ technology allows the EV aggregator to reduce its expected operation cost with respect to the unidirectional charging case.

4. Possible deviations should be compensated in other market mechanisms, e.g., in the balancing market. The incorporation of such market mechanisms in the proposed approach is subject of future research.

\section{Appendix A. Robust Optimization}

This appendix briefly describes how the robust model (13)-(30) described in Section [6 can be derived from the deterministic model (11)-(12) provided in 


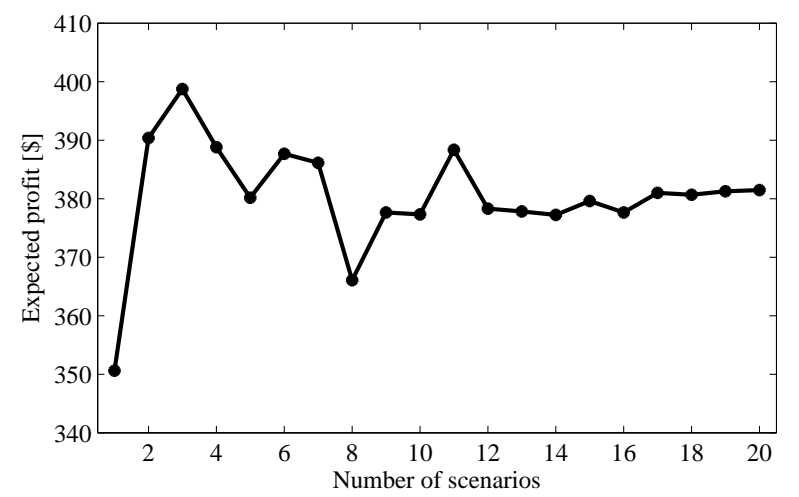

Figure 12: Evolution of the expected profit with the number of scenarios.

500 501 501 model:

$$
\min _{x_{j}, \forall j}
$$

$$
\sum_{j} c_{j} x_{j}
$$

subject to

$$
\begin{aligned}
& \sum_{j} a_{i j} x_{j} \leq b_{i} \quad \forall i \\
& \sum_{j} d_{k j} x_{j} \leq e_{k} \quad \forall k \\
& \sum_{j} f_{l j} x_{j}=g_{l} \quad \forall l \\
& x_{j} \geq 0 \quad \forall t .
\end{aligned}
$$

Problem (A.1)-A.5 has some uncertain coefficients. In particular, these coefficients are the independent terms $b_{i}$ in the inequality constraints A.2. In problem (11)-(12), these uncertain coefficients are $P_{t}^{+, \max }, P_{t}^{-, \max }, E_{t}^{\min }$, and $E_{t}^{\max }$, i.e., the independent terms in constraints (4)-(6). We assume that these coefficients take values within known coefficient bounds, i.e., we know that ${ }_{507} b_{i} \in\left[\underline{b}_{i}, \bar{b}_{i}\right], \forall i$. 
$\min _{x_{j}, \forall j ; v_{i}, \mu_{i}, y_{i}, \forall i}$

$$
\sum_{j} c_{j} x_{j}
$$

subject to

$$
\begin{aligned}
& \sum_{j} a_{i j} x_{j}+\Gamma_{i} v_{i}+\mu_{i} \leq \frac{1}{2}\left(\bar{b}_{i}+\underline{b}_{i}\right) \quad \forall i \\
& v_{i}+\mu_{i} \geq \frac{1}{2}\left(\bar{b}_{i}-\underline{b}_{i}\right) y_{i} \quad \forall i \\
& 1 \leq y_{i} \quad \forall i \\
& v_{i}, \mu_{i}, y_{i} \geq 0 \quad \forall i \\
& \sum_{j} d_{k j} x_{j} \leq e_{k} \quad \forall k \\
& \sum_{j} f_{l j} x_{j}=g_{l} \quad \forall l \\
& x_{j} \geq 0 \quad \forall t .
\end{aligned}
$$

Problem (A.6)-(A.13) is obtained by applying duality and linearization pro${ }_{512}$ prieties [11]. Parameters $\Gamma_{i}$ in constraints (A.7) are included to control the level ${ }_{513}$ of robustness in the model against the uncertain coefficients in each constraint ${ }_{514} i$. Each control parameter $\Gamma_{i}$ can take values between 0 and the number of 515 uncertain coefficients in the corresponding constraint. Since we only consider 


\section{Acknowledgements}

The work of L. Baringo has been partially funded by the Ministry of Science of Spain under CICYT Project ENE2015-63879-R (MINECO/FEDER, UE), the Junta de Comunidades de Castilla-La Mancha under Project POII-2014-012-P, and the Universidad de Castilla-La Mancha under Grant GI20163388.

\section{References}

[1] Z. Li, Q. Guo, H. Sun, Y. Wang, S. Xin, Emission-concerned wind-ev coordination on the transmission grid side with network constraints: Concept and case study, IEEE Transactions on Smart Grid 4 (3) (2013) 1692-1704. doi:10.1109/TSG.2013.2257888.

[2] EV Norway-powered by nature: A guide to Norway, the worlds leading EV marketl, http://www . evnorway.no/ (2016 (accessed April 1, 2016)).

[3] M. Marzband, F. Azarinejadian, M. Savaghebi, J. M. Guerrero, An optimal energy management system for islanded microgrids based on multiperiod artificial bee colony combined with markov chain, IEEE Systems Journal PP (99) (2015) 1-11. doi:10.1109/JSYST.2015.2422253.

[4] M. Marzband, A. Sumper, A. Ruiz-Álvarez, J. L. Domínguez-García, B. Tomoiag, Experimental evaluation of a real time energy management system for stand-alone microgrids in day-ahead markets, Applied Energy 106 (2013) 365-376. doi:10.1016/j.apenergy . 2013.02.018

[5] O. Sundstrom, C. Binding, Flexible charging optimization for electric vehicles considering distribution grid constraints, IEEE Transactions on Smart Grid 3 (1) (2012) 26-37. doi:10.1109/TSG.2011.2168431.

[6] S. Vandael, B. Claessens, M. Hommelberg, T. Holvoet, G. Deconinck, A scalable three-step approach for demand side management of plug-in hybrid vehicles, IEEE Transactions on Smart Grid 4 (2) (2013) 720-728. doi:10.1109/TSG.2012.2213847. 
[7] T. K. Kristoffersen, K. Capion, P. Meibom, Optimal charging of electric drive vehicles in a market environment, Applied Energy 88 (5) (2011) 1940 - 1948. doi:http://dx.doi.org/10.1016/j.apenergy.2010.12.015.

[8] M. González-Vayá, L. Baringo, T. Krause, G. Andersson, P. Almeida, F. Geth, S. Rapoport, EV aggregation models for different charging scenarios, in: Proceedings of the 23rd International Conference on Electricity Distribution (CIRED), 2015.

[9] M. Javadi, M. Marzband, J. L. Domínguez-García, M. Mirhosseini Moghaddam, Non-cooperative game theory based energy management systems for energy district in the retail market considering der uncertainties, IET Generation, Transmission \& Distribution 10 (2016) 2999-3009. doi:10.1049/iet-gtd.2016.0024.

[10] A. J. Conejo, M. Carrión, J. M. Morales, Decision making under uncertainty in electricity markets, Springer, 2010.

[11] D. Bertsimas, M. Sim, The price of robustness, Operation Research 52 (2004) 35-53.

[12] C. Ruiz, A. J. Conejo, Pool strategy of a producer with endogenous formation of locational marginal prices, IEEE Transactions on Power Systems 24 (4) (2009) 1855-1866. doi:10.1109/TPWRS.2009.2030378.

[13] M. Wei, J. Zhong, Optimal bidding strategy for demand response aggregator in day-ahead markets via stochastic programming and robust optimization, in: 2015 12th International Conference on the European Energy Market (EEM), 2015, pp. 1-5. doi:10.1109/EEM.2015.7216732

[14] S. R. Dabbagh, M. K. Sheikh-El-Eslami, Risk assessment of virtual power plants offering in energy and reserve markets, IEEE Transactions on Power Systems 31 (5) (2016) 3572-3582. doi:10.1109/TPWRS.2015.2493182. 
[15] G. Liu, Y. Xu, K. Tomsovic, Bidding strategy for microgrid in day-ahead market based on hybrid stochastic/robust optimization, IEEE Transactions on Smart Grid 7 (1) (2016) 227-237. doi:10.1109/TSG.2015.2476669

[16] R. J. Bessa, M. A. Matos, F. J. Soares, J. A. P. Lopes, Optimized bidding of a EV aggregation agent in the electricity market, IEEE Transactions on Smart Grid 3 (1) (2012) 443-452. doi:10.1109/TSG.2011.2159632.

[17] M. R. Sarker, Y. Dvorkin, M. A. Ortega-Vazquez, Optimal participation of an electric vehicle aggregator in day-ahead energy and reserve markets, IEEE Transactions on Power Systems 31 (5) (2016) 3506-3515. doi:10.1109/TPWRS.2015.2496551,

[18] S. I. Vagropoulos, A. G. Bakirtzis, Optimal bidding strategy for electric vehicle aggregators in electricity markets, IEEE Transactions on Power Systems 28 (4) (2013) 4031-4041. doi:10.1109/TPWRS.2013.2274673.

[19] J. Donadee, M. D. Ili, Stochastic optimization of grid to vehicle frequency regulation capacity bids, IEEE Transactions on Smart Grid 5 (2) (2014) 1061-1069. doi:10.1109/TSG.2013.2290971.

[20] H. Wu, M. Shahidehpour, A. Alabdulwahab, A. Abusorrah, A game theoretic approach to risk-based optimal bidding strategies for electric vehicle aggregators in electricity markets with variable wind energy resources, IEEE Transactions on Sustainable Energy 7 (1) (2016) 374-385. doi:10.1109/TSTE.2015.2498200.

[21] M. González-Vayá, L. Baringo, G. Andersson, Integration of PEVs into Power Markets: A Bidding Strategy for a Fleet Aggregator, in: S. Rajakaruna, F. Shahnia, A. Ghosh (Eds.), Plug In Electric Vehicles in Smart Grids, Springer, 2014, Ch. 9, pp. 233-260.

[22] M. González-Vayá, G. Andersson, Optimal bidding strategy of a plug-in electric vehicle aggregator in day-ahead electricity markets under uncer- 
tainty, IEEE Transactions on Power Systems 30 (5) (2015) 2375-2385. doi:10.1109/TPWRS.2014.2363159.

[23] Z. Xu, Z. Hu, Y. Song, J. Wang, Risk-averse optimal bidding strategy for demand-side resource aggregators in day-ahead electricity markets under uncertainty, IEEE Transactions on Smart Grid PP (99) (2015) 1-1. doi:10.1109/TSG.2015.2477101

[24] L. Baringo, A. J. Conejo, Offering strategy via robust optimization, IEEE Transactions on Power Systems 26 (3) (2011) 1418-1425. doi:10.1109/TPWRS.2010.2092793.

[25] R. Domínguez, L. Baringo, A. Conejo, Optimal offering strategy for a concentrating solar power plant, Applied Energy 98 (2012) 316 - 325. doi:http://dx.doi.org/10.1016/j.apenergy.2012.03.043.

[26] M. Rahimiyan, L. Baringo, Strategic bidding for a virtual power plant in the day-ahead and real-time markets: A price-taker robust optimization approach, IEEE Transactions on Power Systems 31 (4) (2016) 2676 - 2687. doi:10.1109/TPWRS.2015.2483781.

[27] S. A. Gabriel, A. J. Conejo, J. D. Fuller, B. F. Hobbs, C. Ruiz, Complementarity modeling in energy markets, Springer, 2013.

[28] J. Contreras, R. Espinola, F. J. Nogales, A. J. Conejo, ARIMA models to predict next-day electricity prices, IEEE Transactions on Power Systems 18 (3) (2003) 1014-1020. doi:10.1109/TPWRS.2002.804943

[29] The ILOG CPLEX, http://www.ilog.com/products/cplex/ (2016 (accessed April 1, 2016)).

[30] E. Castillo, A. J. Conejo, P. Pedrega, R. García, N. Alguacil, Building and solving mathematical programming models in engineering and science, Wiley, 2001. 
[31] ISO New England, US, http://www.iso-ne.com/ (2016 (accessed April 1, 2016)).

[32] R. E. Rosenthal, GAMS, A user's guide, GAMS Development Corporation, 2012.

[33] J. M. Morales, S. Pineda, A. J. Conejo, M. Carrion, Scenario reduction for futures market trading in electricity markets, IEEE Transactions on Power Systems 24 (2) (2009) 878-888. doi:10.1109/TPWRS.2009.2016072. 